\title{
NARRATIVAS EXPANDIDAS ENTRE LA TRADICIÓN Y LA INNOVACIÓN: CONSTRUYENDO EL UNIVERSO TRANSMEDIAL DE EL MINISTERIO DEL TIEMPO
}

\author{
Concepción CASCAJOSA VIRINO \\ Universidad Carlos III de Madrid \\ ccvirino@hum.uc3m.es
}

\section{Juan Pedro MOLINA CAÑABATE}

Universidad Carlos III de Madrid jpmolina@hum.uc3m.es

\footnotetext{
1 Transmedialidad y ficción televisiva. Narrativas entre la oportunidad y la necesidad El actual ecosistema mediático vive un periodo de profunda transformación, un proceso que parece haberse acelerado en los últimos años gracias a las nuevas tecnologías. Se podría decir que por su propia dependencia de la tecnología, los medios de comunicación están abocados a una situación permanente de mutación, por lo que cada medio acaba encontrando su lugar en el ecosistema. No hay sustitución, sino relaciones cambiantes de complementariedad. Estas se ajustan a las necesidades y los gustos de sus respectivos lectores/espectadores/usuarios. Esto ha favorecido que los intercambios entre los medios a través de narrativas transmediales sean constantes (algo que la sinergia empresarial de las industrias creativas también considera lo más óptimo). $\mathrm{O}$, explicado de otra manera: hoy se producen más adaptaciones y extensiones narrativas donde se combinan diferentes medios. Pero también porque se generan más relatos que nunca, y la manera en la que estos son consumidos muestran también una inédita versatilidad.

El propósito de este texto es realizar un acercamiento a la cuestión de la transmedialidad en el contexto de la televisión contemporánea, y hacerlo, además, entendiendo que supone tanto una necesidad como una oportunidad. Es necesidad en el sentido de que la fragmentación de las audiencias y el panorama multipantalla lleva sin dilación al medio televisivo a luchar por mantener un espacio de centralidad. Pero también es una oportunidad, como veremos, de establecer una nueva relación con su espectador y, para un determinado tipo de programas que podemos denominar «de culto», de lograr una repercusión e ingresos adicionales. Nuestro estudio de caso elegido es el proceso de expansión transmedial de la serie de televisión El Ministerio del Tiempo (Olivares, 2015-), que, sobre la base de
} 
una exploración previa de las posibilidades de las redes sociales y la interacción con los fans, proyectó una compleja estrategia de expansión narrativa con una sorprendente riqueza (en su combinación de nuevos medios y medios tradicionales) y coherencia (en la configuración de rasgos identitarios muy reconocibles por los espectadores habituales).

Antes de pasar a analizar este proceso de expansión transmedial, merece la pena recordar los retos que este nuevo panorama suponen para el análisis. El tradicional marco comparativo de las relaciones cine-televisión dentro de los estudios sobre adaptación se había mostrado como un territorio tan fértil como necesitado de una clara reorientación para pasar de ocuparse de la fidelidad a la exploración del concepto de Bahktin de intertextualidad (Leitch, 2008: 63).

Desde este punto de redefinición, Bruhn, Gjelsvik y Frisvold Hanssen (2013: 4-5) plantearon que en los estudios sobre adaptación la cuestión de la fidelidad convivía con otras cuatro grandes tendencias: 1) La apertura de los estudios de una variedad más amplia de relaciones entre medios más allá de la habitual relación entre novela y cine; 2) la consideración de la adaptación como proceso que tiene lugar en múltiples niveles en lugar de una relación cerrada; 3) la superación de la consideración de los procesos en un sentido único para entenderlos como bidireccionales y dialógicos, y, 4) la incorporación de marcos teóricos globales, como el de la intermedialidad.

Sin embargo, tal y como plantea Virginia Guarinos, los nuevos procesos de hibridación hacen más pertinente la exploración de estos desarrollos a través del concepto de la transmedialidad:

Es la que imbrica medios de comunicación con sus respectivos sistemas sígnicos y mundos virtuales. Este uso de procedimientos y elementos sígnicos de otros medios ya no puede ser entendido como apropiación indebida, ni como imitación ni como falta de recursos propios de un determinado medio. Lo trans es ya hoy una colaboración y mezcla de diversos modos en interacción llegándose al borrado de pertenencia y alcanzando un corpus de elementos universales propios y constitutivos de un modo especial de elaborar discursos del hombre postmoderno, sin importarle de dónde extrae y dónde lo emplea en busca de un enriquecimiento global, en algunos casos como fuente de una ignorancia global, en tanto que no siempre el uso de elementos de otros medios son pertinentes, favorecedores o conscientes y más propios de una cultura del caos gratuito, con la vacuidad de uso de los símbolos que ya no lo son, que dejan de serlo fuera de su contexto y paratexto (2007: 17-18).

Es imposible referirnos aquí al complejo panorama (teórico y metodológico) que este nuevo planteamiento supone. Pero nos sirve, en todo caso, la distinción que realizan Pérez Bowie y Pardo García (2015: 10): transficcionalidad (los materiales narrativos sometidos a constantes procesos de metamorfosis), la transmedialidad (el trasvase entre diferentes medios) y la transescritura (el conjunto de operaciones necesarias para la adecuación a los diferentes medios).

Como veremos con nuestro estudio de caso, en la segunda temporada de El Ministerio del Tiempo hay ficciones especialmente aptas para su paso a otros medios, aunque la probabilidad de éxito va a tener mucho que ver con a quién y con qué planteamiento se encargue el proceso de reescritura. Y es que es innegable que este marco conceptual ha comenzado a transcender de manera definitiva el ámbito de los estudios de adaptación tradicionales para insertarse en un proceso de reconfiguración de las relaciones entre creadores y consumidores. No es casualidad que dos de los primeros teóricos en avanzar en las narrativas transmediales, acortadas como transmedia como derivación de su forma 
anglosajona, fueron autores que se habían preocupado de la intersección entre la expansión narrativa y la gradual asunción de más poder por parte de los usuarios: Marsha Kinder, que exploró el concepto de «intertextualidad transmedia» en su libro Playing with Power in Movies, Television, and Video Games. From Muppet Babies to Teenage Mutant Ninja Turtles (1991), y Henry Jenkins, desde sus primeros estudios sobre fans televisivos y cultura participativa (1992) hasta la formulación del concepto de transmedia story-telling (narrativa transmedia) en el contexto de la cultura de la convergencia (2006).

En los años siguientes, transmedia se ha convertido en un término ubicuo, que, como explica Carlos Scolari (2013: 25), tiene que convivir con una larga serie de términos (cross-media, plataforma múltiples, medios híbridos, mercancía intertextual, mundos transmediales, intermedio, etc.) que iluminan diferentes aspectos de una misma experiencia: «Una práctica de producción de sentido e interpretativa basada en historias que se expresan a través de una combinación de lenguajes, medios y plataformas». De esta forma, tal y como indicamos anteriormente, el transmedia ha comenzado a funcionar en esa dualidad entre la necesidad y la oportunidad en el ámbito del medio televisivo, al amparo de la búsqueda de las nuevas audiencias y del esfuerzo, en un panorama cada vez más fragmentado, de captar la atención del espectador y cultivar su fidelización.

Como se desarrolló en un texto previo (Cascajosa, 2015a), toda la historia de la ficción televisiva ha estado marcada por la transmedialidad, en muchas ocasiones con una vocación de mera explotación comercial y sin una estrategia definida y unificada. Eso ha cambiado especialmente cuando este tipo de experiencias ha dejado de ser una mera externalización en forma de mercados secundarios a ser, al menos en parte, departamentos específicos bajo las denominaciones de «transmedia»o «future media». Este tipo de experiencias no solo están determinadas por las nuevas tecnologías y las posibilidades de interacción que posibilitan. Están también basadas en diseños previos y a largo plazo, que tienen en cuenta los diferentes medios por donde se va a producir la expansión narrativa, con una pretensión mayor de que puedan ser consideradas canónicas y con una participación directa del equipo creativo de la serie de televisión. Es aquí donde El Ministerio del Tiempo se presenta como una experiencia transmedial tan representativa como sofisticada.

\section{La expansión narrativa de $\boldsymbol{E l}$ Ministerio del Tiempo}

El Ministerio del Tiempo se estrenó en febrero de 2015 como una de las apuestas más originales de la ficción española de los últimos años: una combinación de fantasía y aventura histórica con grandes dosis de humor. La premisa era la existencia de un ministerio secreto encargado de custodiar unas puertas que permiten viajar por el tiempo, especialmente para evitar que por accidente o de forma premeditada cambie la historia de España. En el primer capítulo, se presenta a los tres nuevos agentes del Ministerio: Alonso de Entrerríos (Nacho Fresneda), un soldado sevillano de los Tercios de Flandes; Amelia Folch (Aura Garrido), una universitaria barcelonesa de 1880; y Julián Martínez (Rodolfo Sancho), un enfermero madrileño de 2014 traumatizado por la muerte en accidente de su mujer. Los tres son reclutados para formar una de las patrullas del Ministerio del Tiempo por Irene Larra 
(Cayetana Guillén Cuervo) y Ernesto Jiménez (Juan Gea), los eficaces lugartenientes del subsecretario del Ministerio Salvador Martí (Jaime Blanch). La premisa argumental fue creada por los guionistas Pablo y Javier Olivares, a modo de compensación por la imposibilidad de llevar adelante proyectos en la televisión española, y también como homenaje a algunos de sus referentes literarios, cinematográficos y televisivos. La oportunidad de hacer la serie realidad llegó tras el éxito de Isabel, creada por Javier Olivares y en donde escribieron tanto Pablo (que fallecería de ELA en noviembre de 2014) como Anaïs Schaaff, guionista catalana que se había convertido en su principal colaboradora en diversas series para TV3.

Tras su estreno, El Ministerio del Tiempo logró de forma inmediata convertirse en una favorita de los críticos de televisión, además de lograr unas audiencias que, sin ser espectaculares, estaban por encima de las logradas por TVE en un periodo de mínimos históricos. Pero la recepción de la serie sorprendió por dos motivos de forma especial. En primer lugar, la atención mediática prestada a la serie fue muy superior a la popularidad que los audímetros decían que tenía, entre otros motivos porque su perfil de audiencia era más especializado y atraía la atención de perfiles que generan discursos en los medios. En segundo lugar, la reacción en redes sociales, fue extraordinaria y generó con enorme rapidez una significativa comunidad de fans (para una revisión de la recepción de la primera temporada, véase Cascajosa, 2015b). Los «ministéricos», como serían llamados, eran activos en redes, creativos y sentían una enorme necesidad de acceder a experiencias complementarias a las de la serie de televisión. Agustín Alonso, Coordinador de Ficción y Proyectos Transmedia de RTVE, encontró una relación directa entre estos dos factores:

Al final El Ministerio del Tiempo ha hecho ruido porque el seguidor de la serie es alguien influyente -explica. Al final el grupo de edad de entre los 35 y los 45 con poder adquisitivo, con estudios universitarios y urbano, es el que hace opinión. No es por establecer una comparación, pero de Mad Men se habla mucho y luego cuando ves los datos te das cuenta de que es un producto elitista, pero como gusta a los críticos y la gente influyente, se convierte en un fenómeno. En El Ministerio del Tiempo hay algo parecido, aunque en este caso la vea más gente. Hay medios online especializados en televisión que intentan sacar artículos con el titular de El Ministerio del Tiempo porque saben que funciona, el seguidor de la serie es alguien que está muy en Internet (citado en Cascajosa, 2016: 65).

David Varona, entonces a cargo de la coordinación de Nuevos Proyectos en RTVE.es, y Pablo Lara, miembro del equipo de Segunda Pantalla y Televisión Social de RTVE.es, explicaron en un texto la motivación de esta estrategia:

Cuando al equipo de transmedia y segunda pantalla de RTVE.es llegaron los primeros esbozos de lo que sería El Ministerio del Tiempo, estuvo claro que había que hacer algo diferente, algo que se adaptara al público potencial que se quería atraer (joven, aficionado a las series y, por qué no, a la ciencia ficción) y algo que, sobre todo, fuese novedoso y divertido. Con este punto de partida, y siempre con el apoyo de un contenido de calidad, en este caso la serie, se decidió crear una estrategia transmedia que permitiese llevar ese universo de más allá del televisor, a aquellos lugares en red donde los fans se movieran con facilidad (Varona y Lara, 2015: 203).

Varona y Lara, junto con el ya citado Agustín Alonso, fueron los responsables de establecer de forma previa al estreno de la serie una compleja estrategia de expansión transmedial, que incluía la web oficial de la serie, el programa de making of Los Archivos del Ministerio, el programa online 
\#LaPuertadelTiempo, el grupo de Whatsapp «Los becarios del Ministerio», para el que nació también el personaje de Aurelio Pimentel, community manager oficial de El Ministerio del Tiempo, el foro de debate histórico en la web de RTVE.es, la cuenta de Facebook, la cuenta de Twitter @MdT_TVE y la cuenta oficial de Instagram, que funcionaba a modo de archivo secreto del Ministerio.

Sin embargo, aunque esta estrategia se demostró como exitosa para movilizar y visibilizar a las comunidades de fans, también puso de manifiesto que quedaba superada por la generación de materiales narrativos alternativos no canónicos que tomaban a El Ministerio del Tiempo como punto de partida. Se trataba particularmente de relatos de fan fiction, parodias y materiales didácticos creados por admiradores de la serie. La fan fiction ha sido estudiada por Henry Jenkins (1992) como uno de los más interesantes mecanismos a través del cual los fans expresan rasgos de identidad en su reconfiguración y manipulación de las narrativas de partida, y El Ministerio del Tiempo no fue una excepción. Relatos sobre equivalentes al Ministerio en otros países, relaciones amorosas entre los protagonistas, tramas donde personajes secundarios pasan a primer plano y cruces con otros universos ficcionales (como Harry Potter, Titanic o El Hobitt) son algunos de los ejemplos de fan fiction que empezaron a surgir a propósito de la serie, y que en el caso de «Guatemala Connection» se convertía en un juego de rol con intencionalidad educativa para estudiantes de secundaria (Maté, 2015) ${ }^{1}$. El Ministerio del Tiempo se mostraba especialmente apta como punto de partida: en el Ministerio podían trabajar centenares de agentes en un momento dado y las posibilidades de los viajes en el tiempo se presentaban como infinitas. De hecho, el propio Javier Olivares ya había comentado que una de las motivaciones de su hermano Pablo a la hora de desarrollar el concepto era la flexibilidad del concepto narrativo del viaje temporal: «¿Por qué hacer una serie de época, pudiendo hacer una serie con TODAS las épocas?» (prólogo de Olivares a Schaaff y Pascual, 2016: 10). A partir de ese concepto narrativo, cualquier espectador activo podía crear un agente con el que identificarse, y mandarlo a una misión a cualquier momento histórico. Y eso sin tener en cuenta las posibilidades combinatorias con otras narrativas de ambientación de época, a las que el cómic Águila coja, parodia de la serie Águila roja, sacó partido con la entrega «El monasterio del tiempo» (Scolari, 2016).

En este sentido, de cara a una segunda temporada, se produjo una importante expansión del universo narrativo de la serie, esta vez ya como complemento a las estrategias de transmedialidad puestas en marcha en la primera temporada. La expansión transmedial de la primera temporada, más volcada en la exploración de canales de comunicación y en favorecer la interactividad del espectador, se modulaba ahora hacia una expansión narrativa. Todos estos relatos pertenecerían al canon de la serie y, además, tanto en contenido como en tono, iban a ser una ajustada prolongación de esta.

El prólogo a esta estrategia fueron una serie de vídeos difundidos de forma previa al estreno de la segunda temporada donde se relacionaba la serie con aspectos de cultura popular y planteaba uno de los aspectos más distintivos de El Ministerio del Tiempo: una elevada referencialidad que tenía

\footnotetext{
${ }^{1}$ Dos listas diferentes de fan fiction sobre El Ministerio del Tiempo se encuentran en las siguientes direcciones: https://www.fanfiction.net/tv/El-ministerio-del-tiempo/ y http://kalelelvigilante.blogspot.com.es/2015/05/puertassecretas-una-guia-de-fanfics-de.html (última consulta, 15-09-2016).
} 
como consecuencia establecer que los universos de los personajes, a pesar del planteamiento fantástico, eran los mismos que los del espectador. Pablo Lara, que en la segunda temporada ocupó el rol de productor transmedia de la serie (una posición novedosa en el contexto de la fícción española), planteó la función de estos vídeos en la relación con el espectador:

\footnotetext{
El contenido transmedia se crea intentando continuar la percepción por parte del espectador de que $E l$ Ministerio del Tiempo sigue activo, a pesar de estar fuera de emisión. Es decir, pensando contenidos a eventos que ocurran entre una temporada creando la sensación de que la institución vive la vida, el día a día, de los espectadores. Por ejemplo, creando un vídeo homenaje al estreno de Star Wars o a la llegada de Marty McFly de Regreso al Futuro, e incluso comentando un debate político (citado en Cascajosa, 2016).
}

Un ejemplo especialmente interesante tuvo lugar el 20 de octubre, cuando se difundió un video que mostraba a Ernesto en la cafetería del Ministerio viendo el tráiler de la nueva película de Star Wars $^{2}$. Sería la antesala para la producción de materiales específicos para una sección de la página web titulada «Intranet», donde a través de vídeos, documentos, fotografías y audios se ampliaba el universo de la serie. Sin duda, porque estarían fuertemente ligados a otras expansiones del universo de la serie que veremos a continuación, los vídeos grabados por diferentes personas tenían especial interés, casi siempre buscando la conexión emocional o el humor. Por ejemplo, el agente Rogelio Buendía, que se hacía pasar por el Cid, utilizaba un móvil para dejar un mensaje de despedida a su mujer; Spinola grababa por error un vídeo mientras intentaba hacerse un selfi; Argamasilla, nuevo agente del Ministerio, grababa por error un vídeo donde mostraba su interés por la aplicación de contactos sexuales Tinder; Pacino dejaba un mensaje en vídeo a su compañero de piso Alonso quejándose de su falta de limpieza; y Velázquez grababa uno de sus habituales estallidos de ira ${ }^{3}$. Todos funcionaban a modo de pastiche de las variedades de vídeos cortos que cualquier usuario habitual de la Red está acostumbrado a ver, y ahí se encontraba precisamente la clave. La propia variedad de los espectadores de la serie establecía diferentes variedades de expansión narrativa que se iban a materializar principalmente en una serie de ficción sonora (Tiempo de valientes), una webserie (Tiempo de confesiones), una novela (El tiempo es el que es) y un capítulo de realidad virtual («El tiempo es tus manos»).

\section{La revitalización de la narración sonora: Tiempo de valientes}

La expansión narrativa de la segunda temporada de El Ministerio del Tiempo, que de una manera más clara intentaba abrazar el concepto de un medio tradicional en su formulación, fue una serie de podcasts titulada Tiempo de valientes: diario sonoro de Julián Martínez ${ }^{4}$. La mayor parte de las narrativas expandidas sobre las series de televisión orientadas al universo digital han adoptado la forma de las denominadas webseries, cuya estructura y propósito suele ser similar: entregas cortas de pocos

\footnotetext{
${ }^{2}$ Vídeo disponible en www.facebook.com/elministeriodeltiempo, con fecha de 20 de octubre de 2015 (última consulta, 1509-2016).

${ }^{3}$ Vídeos disponibles en http://www.rtve.es/television/ministerio-del-tiempo/contenidos-exclusivos/ (última consulta, 1509-2016).

${ }^{4}$ Serie completa disponible en http://www.rtve.es/television/ministerio-del-tiempo/podcast/ (última consulta, 15-09-2016).
} 
minutos de duración que pertenecen al universo de la serie y que desarrollan aspectos laterales de la trama principal, de forma que sus protagonistas son o bien personajes o bien secundarios de limitada entidad. Y, de forma general, estas webseries se difunden como anticipo del estreno de una nueva temporada de la serie o a modo de puente entre dos de ellas, es decir, en un periodo donde no se encuentra en emisión. La forma habitual que adoptan estas narrativas es lo que convierte a Tiempo de valientes en una experiencia tan original.

La ficción sonora, en forma del radioteatro o del serial radiofónico, fue en su momento una de las principales bases para el desarrollo de la ficción televisiva, tanto por el intercambio de profesionales como por el uso de fórmulas dramáticas y procedimientos técnicos (para ampliar la complejidad de la narrativa radiofónica ficción, véase Guarinos, 2009: 217-262, así como otras obras de la autora, como Guarinos, 1999). Y como parte de esta estrecha relación, se encontraron procesos de adaptación entre ambos medios, fundamentalmente a través de ficciones radiofónicas que daban el salto a la televisión. Es el caso de la pionera serie policiaca Dragnet, posteriormente también llevada al cine, otro ejemplo de que cada nuevo medio establece de forma inevitable procesos de intercambio con los que los preceden.

Es verdad que, con el paso de los años, la relevancia de la ficción sonora ha ido decreciendo, con excepciones relevantes como en Reino Unido. Sin embargo, las nuevas tecnologías se han convertido en un espacio de oportunidad para la radio a través de la distribución en línea (los denominados podcast) y el consumo a la carta en dispositivos portátiles, como demuestra el éxito de la serie de no ficción Serial, una derivación del programa This American Life, que contaba por entregas el proceso judicial en torno a un caso de asesinato (para una aproximación a este fenómeno, véase Berry, 2015). Pero Serial es un exponente más de la revitalización de la narración sonora en este nuevo contexto digital. En España, Radio Televisión Española lo ha explotado desde 2009 bajo el paraguas de Ficción Sonora, un espacio sin regularidad donde se dramatizan obras literarias y relatos cinematográficos. El espacio también ha servido de plataforma para la expansión transmedial de dos series de TVE, Carlos, Emperador (con Carlos de Gante, estrenada en septiembre de 2015) y El Ministerio del Tiempo ${ }^{5}$

La producción de Tiempo de valientes, estrenada, a razón de capítulo semanal (con una duración variable de dos minutos y medio y tres minutos y medio, entre el 15 de febrero y el 21 de marzo de 2016) se puede considerar una expansión narrativa basada también en la sinergia entre diferentes ámbitos de RTVE, particularmente la cadena de televisión La Primera de Televisión Española, Radio Nacional (que produce Ficción Sonora) y la web RTVE.es, donde se alojan los contenidos de ambas y todas sus posibles derivaciones transmediales. De esta forma, también se produce un esfuerzo de promoción mutua, de manera que se daba a conocer Ficción Sonora a los espectadores de El Ministerio del Tiempo y a su vez se presentaba un sucedáneo de la serie para los seguidores del espacio de narraciones sonoras. La ficción sonora se encontraba completamente inserta en los procesos de creación de la serie, siendo guionizada por Pablo Lara, productor transmedia en la segunda temporada de la serie, y Agustín Alonso, Coordinador de Proyectos de Ficción Transmedia de RTVE, con la

\footnotetext{
${ }^{5}$ Web de Ficción Sonora disponible en http://www.rtve.es/radio/ficcion-sonora/ (última consulta, 15-09-2016).
} 
supervisión de los guionistas principales de El Ministerio del Tiempo Javier Olivares y Anaïs Schaaff, mientras que la realización del espacio era responsabilidad de Benigno Moreno y Mayca Aguilera, integrantes del equipo de ficciones sonoras de RNE.

Pero el interés de la estrategia no debe obviar que se trató de un esfuerzo para hacer de la necesidad virtud. Durante el proceso de pre-producción de la segunda temporada de El Ministerio del Tiempo, el actor Rodolfo Sancho anunció su fichaje por la serie policiaca de Antena 3 Mar de plástico. Sancho no abandonó El Ministerio del Tiempo, pero la incompatibilidad entre ambos rodajes llevó a desarrollar una subtrama para justificar su ausencia durante varios capítulos. Así, en los minutos finales del capítulo de debut de la segunda temporada de El Ministerio del Tiempo, «Tiempo de leyenda», emitido el 15 de febrero de 2016, Julián decide huir por una de las puertas el Ministerio a un destino desconocido para sus compañeros. Casi inmediatamente después de la emisión del capítulo, Tiempo de valientes tomaba el testigo, confirmando lo que hasta entonces el espectador de la serie solo podía inferir a partir de la conversación que Julián había mantenido con un agente del Ministerio que había sido soldado en varios frentes: el personaje se ha ido a la Guerra de Cuba en 1898, donde espera utilizar sus conocimientos como enfermero para ayudar a las víctimas del conflicto. Tiempo de valientes, como bien indica su subtítulo, adopta la forma de diario, y viene de forma primordial a anticipar el regreso de Julián a la serie. De hecho, este regresa fugazmente en el cuarto capítulo de la temporada, «El monasterio del tiempo», cuando Irene descubre que el subsecretario del Ministerio Salvador Martí está al tanto del paradero de Julián. En la entrega correspondiente del podcast, Salvador Martí le llama para alertarle de que ha sido descubierto y le señala que debe huir a otro sitio. Sin posibilidad de utilizar las puertas, Julián se despide en la quinta entrega de su diario con una carta a su amigo Manuel, donde anticipa el destino trágico del conflicto, y en la sexta y última entrega ya se encuentra rumbo a México. El aviso de Salvador que se escucha en la cuarta entrega del podcast es precisamente como comienza el capítulo séptimo de la segunda temporada de la serie, titulado igualmente «Tiempo de valientes», en la que se muestra la precipitada huida de Julián. Dos meses después, llega a Filipinas, donde será uno de los soldados del sitio de Baler, el episodio histórico que enmarca este doble capítulo, donde su regreso ya es definitivo.

Tiempo de valientes no solo tiene la utilidad de anticipar la narrativa de la serie, también supone una exploración de elementos temáticos muy importantes en la serie. Es el caso, por ejemplo, de la identificación que establece con los vencidos y las víctimas de los conflictos bélicos, especialmente patente en la segunda entrega, donde Julián narra la muerte de un niño de cinco años debido al hambre y las enfermedades. Esta rememoración del carácter trágico de lo español se pone en especial evidencia en la sexta entrega a propósito de «el desastre del 98»: «Desastre también fue el de 1939, y el de la Armada Invencible. Y lo de Fernando VII. Visto lo visto, viajando por el tiempo, parece que España nunca ha dejado de estar en crisis». La Guerra Civil, la Armada Invencible y el paradójico desenlace de la Guerra de la Independencia ya fueron protagonistas de la serie. Por otro lado, la elevada referencialidad de El Ministerio del Tiempo también se encuentra muy presente, con guiños a obras literarias y cinematográficas. En la tercera entrega, el recuerdo de su amor infantil por Los tres 
mosqueteros de Alejandro Dumas sirve a Julián para expresar sus sentimientos de añoranza de Alonso y Amelia, los otros integrantes de la patrulla, mientras que en la cuarta entrega un diálogo de Desayuno con diamantes de Blake Edwards le sirve para reflexionar sobre la inutilidad de cambiar de lugar para huir de sus problemas. Las referencias al fútbol, que son habituales en la serie, reaparecen aquí en la tercera entrega con un guiño a una misión para evitar que España pierda el Mundial de Fútbol en 2010. Y Tiempo de valientes también se refiere al trabajo previo de Rodolfo Sancho como Fernando de Aragón en Isabel (ya referido en una memorable escena de la primera temporada de la serie) cuando en la cuarta entrega Julián cita una de sus frases para añadir (en una ironía metatextual): «No sé por qué pero, mira, de Fernando el Católico siempre me acuerdo de su nombre». Se trata sin duda de un ejemplo de que no solo la continuidad entre Tiempo de valientes y El Ministerio del Tiempo es importante, sino que la consistencia de tono es igualmente relevante para que ambos textos funcionen en sintonía.

\section{La expansión en el entorno digital: Tiempo de confesiones}

El esfuerzo de expansión narrativa de Tiempo de valientes tuvo continuidad casi inmediatamente con Tiempo de confesiones ${ }^{6}$. Si las seis entregas de Tiempo de valientes se fueron estrenando sucesivamente entre el primer capítulo de la segunda temporada y el regreso de Julián en el capítulo doble (séptimo y octavo) «Tiempo de valientes I», Tiempo de confesiones tomó el testigo tras «Óleo sobre tiempo», la novena entrega de la temporada, para continuar durante cuatro semanas, concluyendo el 19 de mayo de 2016, apenas unos días antes de la emisión del último capítulo de la temporada. El punto de partida de Tiempo de confesiones, escrita y dirigida por Pablo Lara (con la supervisión de Javier Olivares y Anaïs Schaaff), se encuentra en una escena de «Óleo sobre tiempo» en la que Angustias encuentra una cámara de vídeo olvidada en la cafetería del Ministerio. En la primera entrega de Tiempo de confesiones, descubrimos que Angustias no ha encontrado al propietario, por lo que decide utilizarla para hacer pequeñas grabaciones. De una manera evidente, nos encontramos, como en el caso de Tiempo de valientes, un relato en primera persona, solo que en lugar de una narración sonora se trata en este caso de una narración audiovisual que remite directamente a uno de los formatos más populares de Internet, el video-blog. De hecho, así es identificada Tiempo de confesiones en los anuncios del estreno de cada entrega, «el Vblog de Angustias». Tiempo de confesiones también remite a una de las subtramas menores de esta temporada, la existencia de un hijo no conocido por él de Ernesto que resulta ser un youtuber de éxito con el nombre de Nexus 6 (una referencia al clásico de ciencia ficción Blade Runner). Pero a la vez se configura como una inversión casi irónica al resultar Angustias la protagonista: veteranía frente a juventud, intimidad frente a exhibicionismo, reflexión frente a superficialidad.

Como indicamos anteriormente, la mayor parte de las webseries derivadas de series de televisión están protagonizadas por nuevos personajes o personajes ya existentes en la narrativa de partida pero

\footnotetext{
${ }^{6}$ Webserie Tiempo de confesiones disponible en: http://www.rtve.es/television/ministerio-del-tiempo/webserie/ (última consulta, 15-09-2016).
} 
menores. En el caso de Angustias, se trata del segundo caso, pero con una serie de aspectos añadidos. Angustias, secretaria de Salvador y viuda de un antiguo agente del Ministerio, es un referente de serenidad, competencia y compromiso en el contexto dramático de la serie, sirviendo a menudo como confesora de los problemas de los protagonistas. Pero ella también tiene sus propias confesiones que hacer, aunque, debido a su carácter reservado y su soledad, el destino de ellas van a ser estas grabaciones que se presentan al espectador a modo de metraje encontrado. No es casualidad que en la primera de las cuatro entregas, todas de poco más de un minuto y medio de duración, Angustias haga referencia a su encuentro con Napoleón en el capítulo «El monasterio del tiempo», donde por una vez logra protagonismo al ser pieza clave en la resolución de la misión. Angustias se presenta como una «simple secretaria», pero de nuevo la perfecta integración de Tiempo de confesiones en El Ministerio del Tiempo se pone de manifiesto con la exploración de un tema recurrente en la serie. A pesar de la presencia de los grandes nombres de la Historia, son otros los verdaderamente importantes: «Salvador muchas veces no ha dicho que los héroes anónimos son los que hacen que progrese la sociedad». Angustias muestra así sus propias impresiones sobre los acontecimientos del capítulo emitido de forma paralela a Tiempo de confesiones, como los conflictos de Diego de Velázquez en «Óleo sobre tiempo» (entrega 1), el destino trágico de la vampira del Raval en «Separadas por el tiempo» (entrega 2), la ignorancia de Lombardi en «Tiempo de lo oculto» (entrega 3) y el desenlace de la boda en «Hasta que el tiempo nos separe» (entrega 4). Pero sus reflexiones también aluden a acontecimientos previos en la serie, como la devoción a su deber del agente Buendía en el capítulo «Tiempo de leyenda» (entrega 1) o el conflicto de Salvador con el antiguo agente Leiva en el capítulo de la primera temporada «Tiempo de venganza» (entrega 2). La narrativa de los perdedores tan presente en la serie tiene su eco aquí con una referencia a la cultura popular (un aspecto omnipresente en la serie, como ya indicamos anteriormente) cuando en la segunda entrega Angustias esté viendo por televisión la interpretación de Luis Eduardo Aute de su canción «Al alba», dedicada a los últimos fusilados del franquismo.

A nivel tonal, uno de los aspectos más destacados de Tiempo de confesiones es la enorme distancia que la separa de los vídeos introductorios al estreno de la nueva temporada que comentamos anteriormente. Frente al humor, Tiempo de confesiones adopta por momentos un carácter reflexivo y por momentos pesimista, como cuando en la primera entrega Angustias manifiesta su temor a ser olvidada completamente. Quizás a ello contribuya que Tiempo de confesiones es también un vehículo de lucimiento de la actriz Francesca Piñón, prestigiosa actriz catalana que ha elevado a Angustias más allá de su carácter secundario dentro del universo de la serie y aquí puede brillar a través de lo que en esencia son cuatro monólogos sobre la visión del mundo de su personaje. Las confesiones de Angustias finalizan, precisamente, cuando sus reflexiones son interrumpidas por Irene, que bromea con ella, en otro guiño metatextual, con la posibilidad de contar sus propias desventuras y lograr un seguimiento de fans, que se llamarían «ireners» o «angustiers», una referencia directa a las seguidoras de su personaje, que sin embargo adoptaron su nombre de pila al denominarse «cayetaners». De alguna manera, este final sirve de reflejo del doble carácter de Tiempo de confesiones, una expansión de la 
narrativa para los fans de la serie y a la vez demostración del afecto por un personaje secundario y la actriz que lo interpreta.

\section{Regreso al futuro: el capítulo de Realidad Virtual «El tiempo en tus manos»}

La siguiente expansión transmedial de El Ministerio del Tiempo durante su segunda temporada que vamos a reseñar en este texto es el capítulo de Realidad Virtual «El tiempo en tus manos». Se trata de una innovadora experiencia que aprovecha el reciente desarrollo de la realidad virtual a través de dispositivos móviles, por lo que «El tiempo en tus manos» suponía a la vez una estratega promocional y una exploración de un lenguaje en desarrollo. En este caso, el capítulo fue desarrollado de forma conjunta por el Departamento de Nuevos Proyectos de RTVE y la productora Future Lighthouse, con la particularidad de ser promocionado como el primer capítulo de una serie de televisión realizado en forma de Realidad Virtual. Sin embargo, este dato debe contextualizarse, ya que realmente «El tiempo en tus manos» se presenta como una experiencia alternativa a la serie de televisión, no parte de la misma. El título de «El tiempo en tus manos» explica su intencionalidad de servir como vehículo de interacción, además de homenajear la película The Time Machine, una adaptación de la novela de H. G. Wells, y estrenada en España con el título El tiempo en sus manos (el segundo capítulo de la segunda temporada de la serie también recibió este título). Pablo Lara fue co-director y co-guionista del capítulo, compartiendo esas responsabilidades respectivamente con dos profesionales de Future Lighthouse, Nicolás Alcalá y Mariena Ruiz.

«El tiempo en tus manos» se centra exclusivamente en ofrecer una experiencia de interacción que materializa la fantasía de un espectador de la serie de convertirse en un agente del Ministerio. Esta idea (la llamada a la interacción a través de la inserción en el universo diegético) ya se encontraba presente en una de las primeras expansiones narrativas de la serie en la primera temporada. En el grupo de becarios del Ministerio operado a través de la aplicación móvil WhasApp se difundían unos vídeos donde Ernesto e Irene, cumpliendo una función similar a la que desarrollan en la serie, adiestran a nuevos agentes. Destacan dos aspectos clave. En su concepción de la experiencia de consumo ideal con el dispositivo Samsung Gear VR, supone una vivencia de inmersión en el universo del Ministerio tanto a nivel visual en 3D como sonora, aunque existe la posibilidad de ver el capítulo de forma convencional en un móvil. En segundo lugar, de forma complementaria a lo anterior, el aislamiento ideal del espectador a la hora de consumir «El tiempo en tus manos» potencia no solo la experiencia de inmersión, sino también la credibilidad de la misma. El universo representado no funciona únicamente de forma frontal, sino en 360 grados, lo que permite al usuario configurar la experiencia del visionado a través de sus movimientos de cabeza. Estos movimientos de cabeza también permiten realizar diferentes acciones, sobre las que se basa la experiencia de interacción.

En este caso, la trama de «El tiempo en tus manos» es la llegada de un nuevo agente al Ministerio, que es recibido por Ernesto y acompañado al despacho de Salvador, donde realizará una serie de tests para poner a prueba sus conocimientos de la serie. En uno de los vídeos sobre la creación del capítulo, la co-guionista Mairena Ruiz indicó que con esta modalidad se piensa una historia, pero sobre la base 
de que la mirada del espectador configura la plasmación visual de la misma. Sin embargo, la posibilidad de acción del usuario es limitada y las acciones que debe realizar se indican de una manera clara, a veces con la inclusión de otro personaje que da instrucciones o señala objetos ${ }^{7}$. En el caso de «El tiempo en tus manos», la acción se limita a una prueba como nuevo agente y a los escenarios del Ministerio. En este sentido, tiene mucho más interés desde el punto de vista de la experiencia estética (sentirse dentro del Ministerio) que del envolvimiento narrativo. Pero a pesar su carácter experimental, «El tiempo en tus manos» muestra la misma coherencia con el universo de la serie de televisión que el resto de expansiones transmediales que hemos reseñado anteriormente. Es el caso del guiño al espectador que supone la aparición del co-creador Javier Olivares y la co-guionista Anaïs Schaaff como agentes que realizan el test junto con el usuario, siendo ambos populares entre los seguidores por su aparición en los documentales de «cómo se hizo» que se emiten antes y después de los capítulos.

\section{La expansión narrativa como «capítulo perdido»: la novela El tiempo es el que es}

La novela El tiempo es el que es demuestra que en el caso de El Ministerio del Tiempo, las experiencias transmediales de la serie han adoptado tanto formas nuevas como tradicionales. La novela El Ministerio del Tiempo es un exponente más de la estrecha relación que tienen en el momento actual la industria literaria y la televisiva en el contexto español. Estas se basan en un flujo entre ambos medios: de la literatura a la televisión a través de las adaptaciones y de la televisión a la literatura a través de las novelizaciones. El éxito de estos intercambios para la industria editorial es evidente: nuevas ediciones de novelas se ponen en circulación con los rostros televisivos en portada y una parte muy sustancial de series de éxito cuenta con una novela. La relación entre los universos diegéticos suele ser en este segundo caso variable, lo que demuestra la propia flexibilidad de la forma. En el sentido tradicional, una novelización supone la traslación al lenguaje literario de la trama de un relato audiovisual, lo que fundamentalmente supone la incorporación de la subjetividad de los personajes y de mayor información contextual. Sin embargo, aunque esta es la forma preeminente en el caso de películas, este tipo de traslación no es la más habitual en el caso de las series de televisión, con alguna excepción relevante como las dos novelas escritas por Salva Rubio sobre las sendas temporadas producidas de la serie policiaca El Príncipe (2014 y 2016). La modalidad más habitual de novelización se plantea como una expansión narrativa del universo televisivo. A veces, se trata de revelar acontecimientos que ocurrieron antes del comienzo del programa, lo que podemos denominar narrativas de origen. Un ejemplo es Seis hermanas. Los años de la inocencia (2015), que muestra la juventud de la madre de las protagonistas de la serie unos 30 años antes del comienzo de la misma, aunque en otros casos, como Toda una vida (2015), se trata de presentar la juventud de la pareja protagonista de Cuéntame cómo pasó.

En otros casos, las novelas derivadas de series de televisión presentan tramas con personajes de la serie que pueden haber tenido lugar pero que no se han mostrado a los espectadores, lo que es

\footnotetext{
7 Video disponible en http://www.rtve.es/alacarta/videos/el-ministerio-del-tiempo/ministerio-del-tiempo-asi-se-hizotiempo-tus-manos/3553468/ (última consulta, 15-09-2016).
} 
especialmente apto para series con planteamientos de aventuras o basados en tramas episódicas. Es esta la forma adoptada por El tiempo es lo que es (2016), que se editó mientras la segunda temporada de la serie se encontraba en emisión. Como en la mayor parte de las novelizaciones, los autores de $E l$ tiempo es el que es eran guionistas de la serie, en este caso Anaïs Schaaff y Javier Pascual, con Javier Olivares asumiendo labores de edición. La razón de esta estrecha vinculación entre los autores de una novelización y de la serie tiene sentido por varios motivos. La novela debe ser coherente con la caracterización de los personajes, así como mantener continuidad con las tramas ya conocidas por el espectador habitual, que es el lector predilecto de este tipo de productos. Pero hay un factor más: las novelas basadas en relatos audiovisuales suelen tener primacía de los diálogos por encima de las descripciones, y la manera de hablar de los personajes tiene que estar en consonancia con la manera en la que se expresan en la serie. Pero hay otros aspectos en los que la novela de El Ministerio del Tiempo es una experiencia relativamente novedosa. Hay un motivo evidente por el que la mayor parte de las novelizaciones se desarrollan en el pasado: para establecer una separación lo suficientemente amplia entre la narrativa de la novela y de la serie como para que la primera no comprometa a la segunda.

Sin embargo, aquí se opta por establecer un marco temporal claro e inserto en la mitad de la segunda temporada, concretamente entre los capítulos 19 («Tiempo de lo oculto») y 20 («Hasta que el tiempo nos separe»). En términos del desarrollo de tramas, en ese punto el personaje de Julián ya ha vuelto de su auto-impuesto exilio y Amelia ha descubierto (en la escena de final «Tiempo de lo oculto») que en la fotografía antigua en la que aparecía con Julián y un bebé, este último ha desaparecido, lo que parece relacionado con el corto romance que vivió en la primera parte de la temporada con Pacino. Por su parte, Alonso se encuentra en medio de su conflictivo romance con la abogada Elena, idéntica físicamente a su mujer Blanca. Este marco da la resonancia emocional a las aventuras que van a vivir en la novela, sirviendo de prólogo al desarrollo dramático que se va a vivir en el capítulo 20 «Hasta que el tiempo nos separe», en el que Amelia confiesa a Julián su romance con Pacino, lo que parece cerrar las puertas a su relación, y Alonso es abandonado por Elena cuando esta descubre su peligroso estilo de vida. La otra manera en la que la narración de El tiempo es el que es se relaciona con la de la serie de televisión es a través del personaje de Lola Mendieta (Natalia Millán), recurrente en la serie como antagonista. Antigua agente del Ministerio que lo traiciona para comerciar con arte y alterar la Historia en momentos puntuales, la última aparición de Lola se produce en el sexto capítulo de la segunda temporada, cuando descubre que viajar en el tiempo utilizando el procedimiento de una empresa norteamericana, Darrow, le ha producido un cáncer. Lola aparece al comienzo y al final de la novela, primero en lo que parecen ser sus últimos días de vida en un hospital de Madrid y después en una versión más joven, cuando colaboraba con la Resistencia francesa durante la II Guerra Mundial. En su prólogo del libro, Javier Olivares (en Schaff y Pascual, 2016: 14) explica que su relevancia en la novela sirve para compensar un tratamiento no del todo ideal en la serie: «Un personaje que sin duda, si los medios de producción hubieran sido otros, habría merecido un mejor desarrollo. Esta novela es un homenaje a ella». 
Como novela, El tiempo es el que es adopta una estructura particular, ya que narra tres aventuras de los personajes en lugar de presentar una trama única. Al parecer, por lo que cuenta Javier Olivares en el prólogo, eso es debido al reciclaje que se realiza de capítulos no producidos para cada una de las tres aventuras, uno perteneciente a la primera pero rechazado en su momento por TVE por no incluir ningún personaje histórico de entidad y los otros dos por la imposibilidad de ser realizados debido a los medios de producción. De esta forma, en la primera entrega la patrulla se desplaza al año 808 para rescatar a un antiguo agente del Ministerio llamado Elías Sotoca, aunque en realidad es una trampa para atraer a otros agentes con los que paliar su soledad. En la segunda, la patrulla descubre que la puerta que han utilizado para salir del 808 les ha llevado a Cartagena de Indias y deben regresar, a pesar de que en el barco de vuelta se produce un robo que deberán resolver. Por último, en la tercera entrega deben completar la «Operación Mincemeat» para garantizar que el desembarco de los Aliados en Sicilia durante la II Guerra Mundial sea un éxito, mientras que una joven Lola Mendieta debe ser rescatada junto con Ernesto tras ser atrapados por la Gestapo y enviados a un campo de concentración. Esta estructura permite emular uno de los aspectos más característicos de la segunda temporada, esto es la alteración de géneros de capítulo a capítulo. De esta forma, la primera parte de El tiempo es el que es se ajusta a una aventura de rescate dramático, la segunda a una investigación detectivesca y la tercera a un relato de espionaje. Y como siempre ocurre en la serie, el trasfondo histórico sirve para dar resonancia a las aventuras de los personajes, que en los tres casos además reflejan la sensibilidad feminista de la serie: en la primera entrega Alonso salva a una campesina de ser violada y Amelia es casi obligada a casarse con el villano, mientras que en la segunda los personajes conocen en su juventud a Catalina de Erauso, la conocida como «Monja Alférez», que en el siglo XVII desafió a los cánones de la época para vivir como mercenario en las colonias americanas. Por último, la tercera aventura da contexto al personaje de Lola Mendieta, convertida aquí en heroica luchadora contra el nazismo. Si la primera entrega hace recordar por su uso del concepto del impostor a los capítulos «Tiempo de pícaros» y «Tiempo de leyenda», el tercero recupera el tema del papel desempeñado por España durante la Segunda Guerra Mundial del capítulo «Cómo se reescribe el tiempo».

Pero la perfecta sincronía entre la novela y la serie de televisión se pone de relevancia en otros muchos aspectos de El tiempo es el que es, especialmente a través de las referencias directas a diferentes episodios de la serie. Dos de estas referencias se centran en el capítulo final de la primera temporada «Tiempo de leyenda»: primero Alonso recuerda haber ayudado a su esposa Blanca a huir del maltrato de su nuevo marido con una idea sacada del Don Juan Tenorio, y luego Julián se enfurece recordando el trágico destino de Federico García Lorca cuando escucha en la aventura final que un joven ha sido encontrado en una cuneta, probablemente asesinado por homosexual. Y también está la recurrencia del tema de los vencidos, especialmente patente en las referencias a la Guerra Civil y particularmente a la referencia que se hace al poeta Miguel Hernández como una de «las víctimas de la Historia» (Schaaff y Pascual, 2016: 93). Y también a los héroes anónimos, como John Roberts Martínez, que se sacrifica para completar la «Operación Mincemeat» en la tercera aventura. Pero es en el tono donde la coherencia entre El tiempo es el que es y la novela se muestra más directa. La 
inteligencia de Amelia, el talante valeroso de Alonso y el distanciamiento irónico de Julián son aspectos clave en la caracterización de los personajes en la novela. Y también son recurrentes los elementos de referencialidad en la novela, con guiños a las series Macgyver (148), Se ha escrito un crimen (135) y Juego de tronos (163), además de películas como El motín del Caine (133) y Sor Citroën (157). Por último, la pasión futbolística de los artífices de la serie y especialmente por el Atlético de (algo que Olivares comparte con el co-autor de la novela Javier Pascual, tal y como indica en la página 12 del prólogo), se manifiesta en las referencias directas al entrenador actual de este equipo, Diego «Cholo» Simeone (105 y 248), la segunda para rememorar su frase más conocida: «Partido a partido». La última entrega finaliza precisamente en un pub de fútbol inglés, donde Alonso bebe cerveza y habla de este deporte con el hijo de John Roberts Martínez después de la Segunda Guerra Mundial.

\section{Conclusiones}

Como producto transmediático, El Ministerio de Tiempo ha experimentado un desarrollo en el aspecto tecnológico, que ha conllevado también mayor profundidad en la trama argumental y en el crecimiento de los personajes. El escenario se abre para el espectador, que puede verlo (realidad virtual) o puede imaginarlo (podcast), ofreciéndole más posibilidades de implicación. Como explica el consultor de comunicación especialista en procesos transmedia Diego Rivera, el espectador asiste al desarrollo de la historia como un testigo privilegiado o co-partícipe de la misma y se sumerge hasta la profundidad que él desee según la recompensa emocional o intelectual que reciba.

Esta inmersión (privada, casi íntima) en el océano argumental y la respuesta emocional que provoca se retroalimenta, a su vez, y tal como hemos visto más arriba, de la creciente sensibilidad de los últimos capítulos de la segunda temporada (feminismo, antibelicismo, nueva percepción de la soledad y del amor). Todos estos ingredientes hacen de El Ministerio del Tiempo una experiencia que traspasa la pantalla.

\section{Bibliografía}

AlCALÁ, N. - LARA, P. (2016): «El tiempo en tus manos», en http://www.rtve.es/television/ministeriodel-tiempo/vr/ (última consulta, 15-09-2016).

BERRY, R. (2015): «A Golden Age of Podcasting? Evaluating Serial in the Context of Podcast Histories», Journal of Radio \& Audio Media, 22/2, pp. 170-178.

Bruhn, J.; GJelsvik, A.; Hanssen, E. F. (2013): «There and Back Again”: New Challenges and New Directions in Adaptation Studies», en J. Bruhn, A. GJELsviK y E. F. Hanssen, eds., Adaptation Studies: New Challenges, New Directions. Londres, Bloomsbury, pp. 1-16.

CASCAJOSA VIRINO, C. (2015a): «Transmedialidad y ficción televisiva en Estados Unidos: de lo analógico a la realización digital», en J. A. PÉREz BowIE y P. J. PARDo GARcía, eds., Transescrituras audiovisuales. Madrid, SIAG Pigmalión, pp. 111-126. 
, ed. (2015b): Dentro de «El Ministerio del Tiempo»: el libro sobre la serie que ha revolucionado la Televisión en España. Alcalá de Henares, Léeme Libros.

_ (2016): «Buscando al espectador serial desesperadamente: la nueva investigación de audiencias y la serie El Ministerio del Tiempo», Revista Dígitos, 2, pp. 53-70.

GuARINOS, V. (1999): Géneros ficcionales radiofónicos. Madrid, Mad.

- (2007): «Transmedialidades: el signo de nuestro tiempo», Comunicación, 5, pp. 17-22.

- (2009): Manual de narrativa radiofónica. Madrid, Síntesis.

Jenkins, H. (1992): Textual Poachers: Television Fans and Participatory Culture. Londres, Taylor \& Francis.

- (2006): Convergence Culture: Where Old and New Media Collide. Nueva York, NYU Press.

Kinder, M. (1991): Playing with Power in Movies, Television, and Video Games. Los Angeles, University of California Press.

LARA, P. (2016): Tiempo de confesiones (webserie), en http://www.rtve.es/television/ministerio-deltiempo/webserie/ (última consulta, 15-09-2016).

LeITCH, T. (2008): «Adaptation Studies at a Crossroads», Adaptation, 1/1, pp. 63-77.

MAté Puig, I. (2015): «Enseñando, que no es poco: El Ministerio del Tiempo en el aula de Historia», en C. CASCAJOSA VIRINO, ed. (2015b), pp. 249-256.

Moreno, B. - Aguilera, M. (2016): Tiempo de valientes: diario sonoro de Julián Martínez, en http://www.rtve.es/television/ministerio-del-tiempo/podcast/ (última consulta, 15-09-2016).

Olivares, J. y P. (2015-): El Ministerio del Tiempo (DVD). Madrid, Divisa.

Pérez Bowie, J.A. - Pardo García, P. J. (2015): «Presentación», en J. A. Pérez Bowie y P. J. Pardo García, eds., Transescrituras audiovisuales. Madrid, SIAG Pigmalión, pp. 9-18.

SchaAfF, A. - PAscual, J. (2016): El tiempo es el que es. Barcelona, Plaza \& Janés.

SCOlARI, C. A. (2013): Narrativas transmedia: cuando todos los medios cuentan. Barcelona, Deusto. - (2015): «Regreso a El Ministerio del Tiempo», en CASCAJOSA VIRINO, ed. (2015b), pp. 227232.

VARONA ARAmburu, D. y LARA TOledo, P. (2015): «"Be ministérico, my friend”: Diseño de una estrategia transmedia», en CASCAJOSA VIRINO, ed. (2015b), pp. 203-210. 